\title{
Improved Correspondence for DTI Population Studies Via Unbiased Atlas Building
}

\author{
Casey Goodlett ${ }^{1}$, Brad Davis ${ }^{1,2}$, Remi Jean ${ }^{3}$, \\ John Gilmore $^{3}$, and Guido Gerig ${ }^{1,3}$ \\ ${ }^{1}$ Department of Computer Science, University of North Carolina \\ 2 Department of Radiation Oncology, University of North Carolina \\ ${ }^{3}$ Department of Psychiatry, University of North Carolina*
}

\begin{abstract}
We present a method for automatically finding correspondence in Diffusion Tensor Imaging (DTI) from deformable registration to a common atlas. The registration jointly produces an average DTI atlas, which is unbiased with respect to the choice of a template image, along with diffeomorphic correspondence between each image. The registration image match metric uses a feature detector for thin fiber structures of white matter, and interpolation and averaging of diffusion tensors use the Riemannian symmetric space framework. The anatomically significant correspondence provides a basis for comparison of tensor features and fiber tract geometry in clinical studies and for building DTI population atlases.
\end{abstract}

\section{Introduction}

Diffusion tensor imaging (DTI) has become increasingly important as a means of investigating the structure and properties of neural white matter. The local diffusion properties of water in the brain can be measured in vivo using diffusion tensor MRI (DT MRI). In brain tissue, water diffuses more easily along myelinated axons which make up the white matter fiber bundles. Acquiring multiple images with different gradient sensitizing directions provides an estimate for the local diffusion tensor at each voxel. The major eigenvector of each tensor corresponds to the direction of the local fiber bundle, and the field of principal eigenvectors can be integrated to produce fiber tracts.

Many approaches have been proposed to analyze DTI in clinical studies. For example, derived scalar properties such as fractional anisotropy (FA), relative anisotropy (RA), or mean diffusivity (MD) of the tensors are often compared in regions of interest drawn by experts. Other methods have characterized the geometry of white matter through tractography, as well as quantitative analysis of tractography [1. However, region of interest approaches require an expert

\footnotetext{
* This work is part of the National Alliance for Medical Image Computing (NA-MIC), funded by the National Institutes of Health through the NIH Roadmap for Medical Research, Grant U54 EB005149. The authors acknowledge support from the NIMH Silvio Conte Center for Neuroscience of Mental Disorders MH064065 as well as grant support from the National Alliance for Autism Research (NAAR) and the BlowitzRidgeway Foundation.
} 


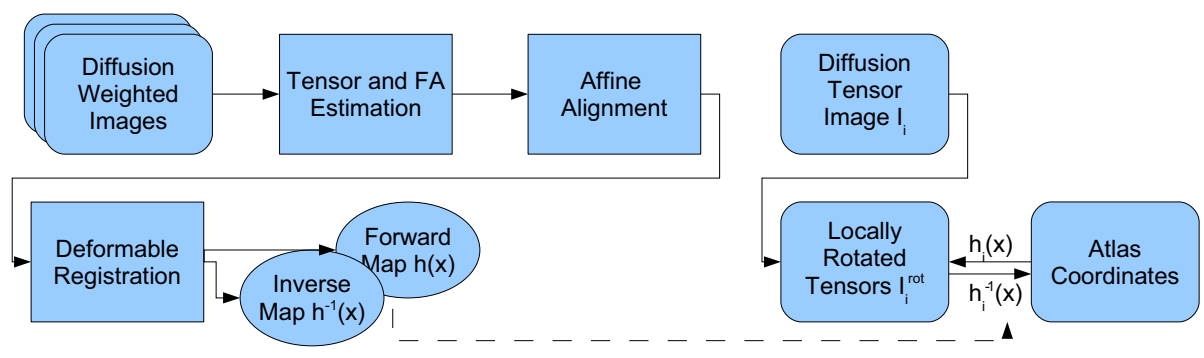

Fig. 1. Flowchart of atlas building process

to segment structures of interest, and inter-subject comparison of tractography lacks the correspondence between fiber tracts needed to make statistical comparisons. Wakana et al. 2] have built a fiber-tract atlas in the form of voxel maps of prior probabilities for major fiber bundles. We propose to build an atlas for tensor images to provide a basis for statistical analysis of tensors, tensor-derived measures, and fiber bundle geometry.

We use the techniques of registration and atlas-building to provide intersubject correspondence for statistical analysis of diffusion data as shown in Figure 1. Our metric for optimizing the registration parameters is based on a structural operator of the tensor volumes. An initial alignment is performed by computing the affine transformation between the structural images and applying the transformation to the tensor volumes. An unbiased, deformable atlas-building procedure is then applied which produces mappings between each subject and a common atlas coordinate system using the method of Joshi et al [3]. We validate our method by showing an improvement over affine registration alone.

\section{Registration}

Several image metrics have been proposed for inter-subject registration of DTI including metrics based on the baseline images and the full diffusion tensors 45]. We propose an intermediate, heuristic solution between using only baseline images and using metrics based directly on the diffusion tensors. Our method is based on a structural operator of the FA image that is more sensitive to major fiber bundles than metrics based only on the baseline images. Given a tensor image $I$ and the corresponding FA image $F A$, the structural operator $C$ is defined in terms of the maximum eigenvalue of the Hessian,

$$
C=\max [\text { eigenvalues }(\mathbf{H})], \text { where } \mathbf{H} \equiv\left(\begin{array}{lll}
F A_{x x} & F A_{x y} & F A_{x z} \\
F A_{y x} & F A_{y y} & F A_{y z} \\
F A_{z x} & F A_{z y} & F A_{z z}
\end{array}\right) .
$$

Figure 2 shows the FA image of a tensor field and the corresponding structural image $C$. Let $h_{i}(x)$ be a mapping which gives the corresponding point in the subject image $I_{i}$ for all $x$ in the domain $\Omega$ of the atlas image $\hat{I}$. Given two 

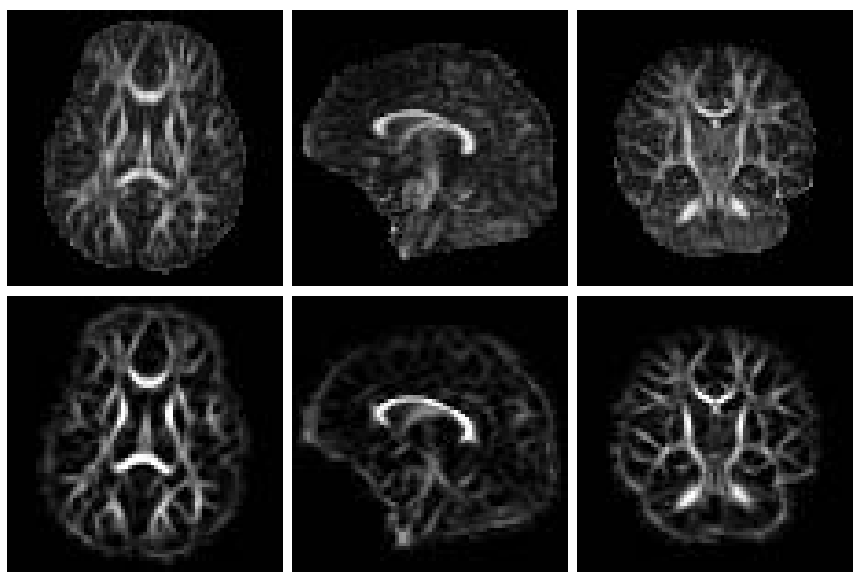

Fig. 2. The top row shows axial, sagittal, and coronal slices of the FA image from a DTI scan of a 1-year old subject. The bottom row shows the result of the structural operator on the FA image taken at $\sigma=2.0 \mathrm{~mm}$. Major fiber bundles such as the corpus callosum, fornix, and corona radiata are highlighted, while the background noise is muted.

images $I_{1}$ and $I_{2}$ the image match functional that is optimized in the registration process is

$$
M\left(I_{1}(x), I_{2}(h(x))\right)=\int_{x \in \Omega}\left[C_{1}(x)-C_{2}(h(x))\right]^{2} d x,
$$

the mean squared error between $C_{1}$ and $C_{2}$.

We use $C$ over existing methods for two main reasons. First, we observe that $C$ is a good detector of major fiber bundles which occur as tubular or sheet-like structures. Callosal fibers form a thin swept U; the corona radiate is a thin fan; the cingulum is a tubular bundle, and $C$ serves as a strong feature detector for all types of these thin structures. Consequently, $C$ optimizes correspondence of fiber tracts better than the baseline image, because $C$ has the strongest response at the center of major fiber bundles, while the baseline image has the strongest signal in the cortico-spinal fluid (CSF). Secondly, we use $C$ instead of a full tensor metric or the FA itself in order to minimize overfitting the diffeomorphic registration by using the same feature for registration that will be used for statistical comparison. The Hessian at a fixed scale is a first step towards basing the registration on a geometric model of the white matter, and future work will investigate a multi-scale approach to computing $C$ to make the measurement dependent only on the local width of the structure.

Using our definition for an image match functional, registration of the images proceeds in two stages. First, a template tensor image is aligned into a standardized coordinate system by affine alignment of the baseline image with a T2 atlas using normalized mutual information. The remaining tensor images are then aligned with the template using an affine transformation and equation 2. In this coordinate system, we can average the tensor volumes to produce an affine atlas. 


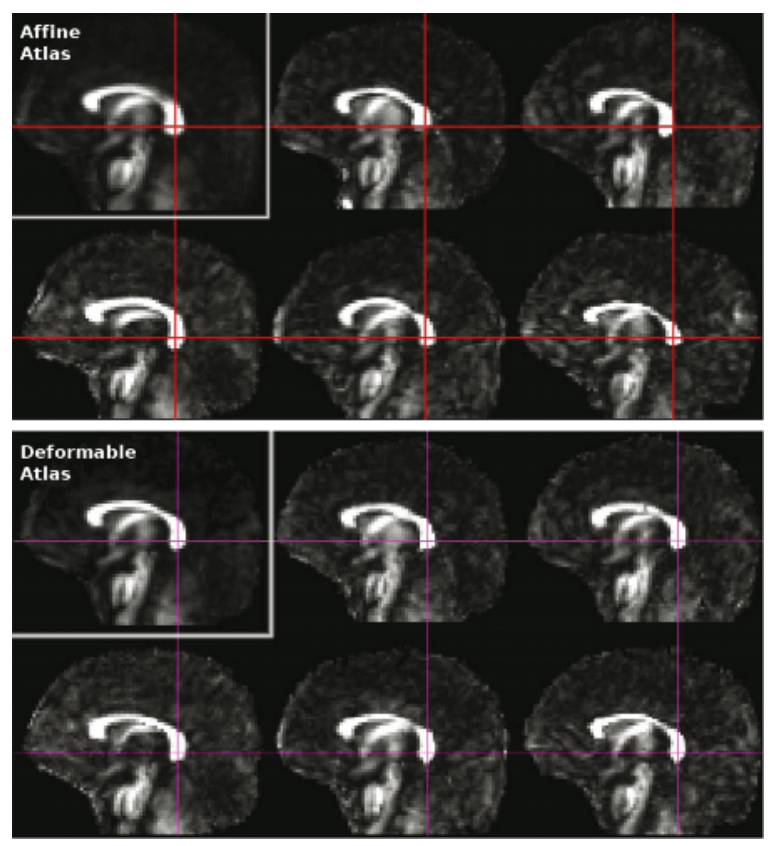

Fig. 3. The top six images show the correspondance of the affine atlas and the five subject images at a point in the splenum of the corpus callosum. Notice the corresponding index in the subject images does not necessarily correspond to the same anatomical location. The bottom six images show the deformable atlas and subject images with the same point selected; Here the atlas provides better anatomical correspondence, and the deformable atlas has sharper structures.

However, the affine registration does not account for the non-linear variability of the white matter geometry, and many of the white matter structures are blurred in the atlas volume. For this reason, we subsequently apply a deformable registration procedure to obtain anatomical correspondence between the population of images $I_{i}$ and a common atlas space $[3$. This procedure jointly estimates an atlas image $\hat{I}$ and a set of diffeomorphic mappings $h_{i}$ that define the spatial correspondence between $\hat{I}$ and each $I_{i}$. Figure 3 shows the improved correspondence attained from the deformable registration. The computed transformations are applied to each tensor volume as described in the next section.

\section{Tensor Processing}

The application of high-dimensional transforms to the DTI volumes must account for the space of valid tensors. The orientation of a diffusion tensor provides a measurement of fiber orientation relative to the anatomical location, and spatial transformations of the tensor fields must account for the reorientation of the tensor. Furthermore, since diffusion tensors are symmetric positive-definite matrices, operations on the images must preserve this constraint. 


\subsection{Spatial Transformations of Tensor Images}

When spatial transformations of diffusion images are performed to align the anatomy of different scans, the tensors must also be transformed to maintain the relationship between anatomy and anisotropy orientation. We use the finite strain approach of Alexander et al. to reorient tensors in a deformation field by decomposing the local linear approximation of the transformation into a rotation and deformation component [6]. The rotation of each tensor is computed by performing singular value decomposition (SVD) on the local linear approximation of the transformation $F$, where $F$ is given by the Jacobian matrix of the deformation field computed by finite differencing. A local linear deformation $F$ is decomposed into a rotation matrix $R$ and a deformation matrix $U$, where

$$
F=U R .
$$

The local transformation of a tensor $D$ is given as

$$
D^{\prime}=R D R^{T} .
$$

\subsection{Interpolation and Averaging of Tensor Images}

The space of valid diffusion tensors does not form a vector space. Euclidean operations on diffusion tensors such as averaging can produce averages with a larger determinant than the interpolating values, which is not physically sensible. Furthermore, operations on diffusion tensors are not guaranteed to preserve the positive-definite nature of diffusion. The Riemannian framework has been shown as a natural method for operating on diffusion tensors, which preserves the physical interpretation of the data, and constrains operations to remain in the valid space of symmetric positive-definite matrices $[7 / 8$. Further simplifications have shown an efficient method for computation using the Log-Euclidean metric 9]. Interpolation and averaging are treated as weighted sums in the Log-Euclidean framework defined as

$$
\hat{D}=\exp \left(\sum_{i=1}^{N} w_{i} \log \left(D_{i}\right)\right),
$$

where log and exp are the matrix logarithm and exponential functions. To produce the atlas tensor volume the deformed tensor volumes with locally rotated tensors are averaged per-voxel using the Log-Euclidean scheme,

$$
\begin{aligned}
I^{\mathrm{rot}}(x) & =R(x) I(x) R(x)^{T}, \\
\hat{I}(x) & =\exp \left(\frac{1}{N} \sum_{i=1}^{N} \log \left(I_{i}^{\mathrm{rot}}\left(h_{i}(x)\right)\right)\right) .
\end{aligned}
$$

\section{Experiments and Results}

Our methodology was tested on five datasets of healthy 1-year olds from a clinical study of neurodevelopment. The images were acquired on a Siemens head-only 


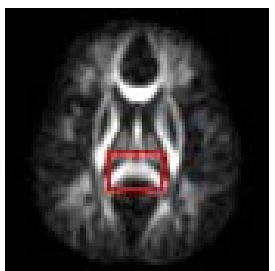

(a) Affine

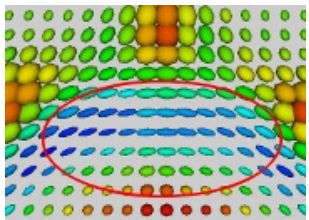

(b) Affine

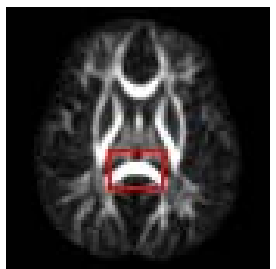

(c) Deformable

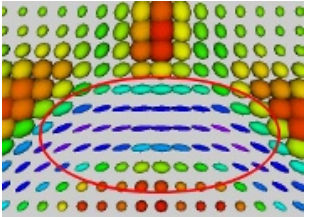

(d) Deformable

Fig. 4. 4(a) and 4(c) show a slice of the FA for the affine and deformable atlases. 4(b) and $4(\mathrm{~d})$ illustrate a subregion of tensors in the splenum of the corpus callosum. Notice that the FA image of the affine atlas is more blurry, and that the tensors in the splenum are more swollen in the affine atlas.

3T scanner. Multiple sets of diffusion weighed images were taken for each subject and averaged to improve signal-to-noise ratio. Each dataset consisted of one baseline image and six gradient direction images with $b=1000 \mathrm{~s} / \mathrm{mm}^{2}$ using the standard orientation scheme. An image volume of $128 \times 128 \times 65$ voxels was acquired with $2 \times 2 \times 2 \mathrm{~mm}$ resolution. Imaging parameters were $\mathrm{TR} / \mathrm{TE}=$ $5200 \mathrm{~ms} / 73 \mathrm{~ms}$.

For each set of diffusion weighted images the diffusion tensors were estimated using the method of Westin et al [10. The structural image $C$ was computed from the FA volume with $\sigma=2.0 \mathrm{~mm}$. Affine and deformable alignment were computed using the methods described in section 2. The warped DTI volumes were averaged to produce an affine atlas and a deformable atlas. Figure 4 shows a comparison between the tensor volumes of the two atlases.

Tractography was performed in the atlas space, and the tract bundles were warped to each subject image using $h_{i}$. Figure 5 shows the results of tractography in the atlas and the corresponding warped fibers in two subject images.

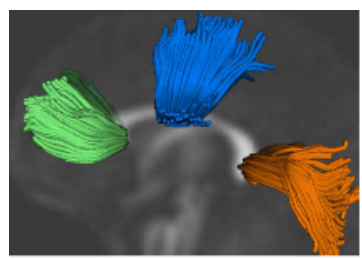

(a) Atlas

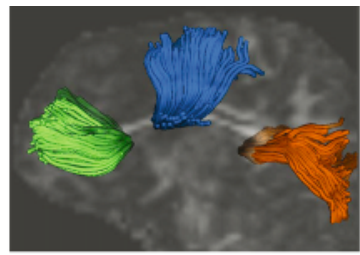

(b) Image 1

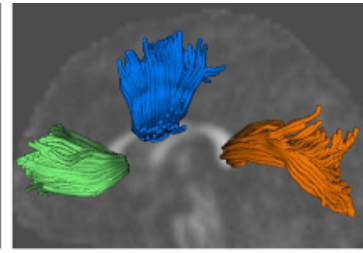

(c) Image 2

Fig. 5. Fibers traced in the corpus callosum of the atlas (a) are mapped to corresponding locations in the subject images (b) (c) despite pose and shape changes.

\section{Validation}

Visual inspection of tractography in the atlas volume shows an initial qualitative validation that the registration and averaging methods provide anatomically sensible results. Histogram comparisons of derived tensor measures in the affine and 


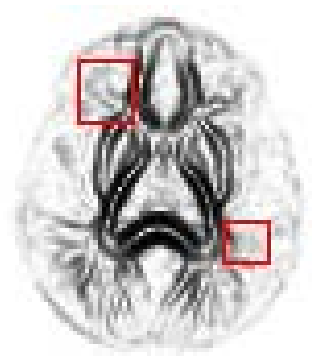

(a) Affine

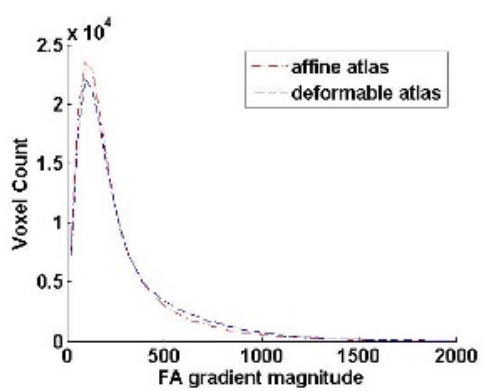

(c) Gradient Magnitude Histogram

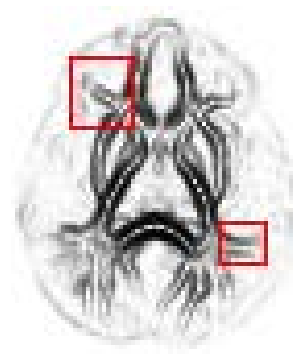

(b) Deformable

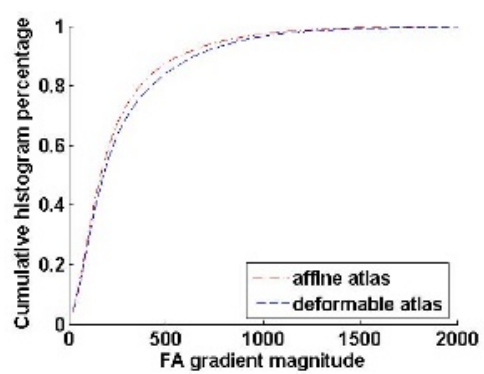

(d) Cumulative Histogram

Fig. 6. (a) and (b) show an axial slice of the gradient magnitude image. Notice the structures in the marked regions which are visible in the deformable atlas, but not the affine atlas, and the increased sharpness of the splenum of the corpus callosum. Figures (c) and (d) show the histogram and cumulative histogram of gradient magnitude intensities in the whole brain.

diffeomorphic atlas show an initial quantitative validation of the improvement of the deformable registration over affine registration alone. The gradient magnitude of the FA was measured in the whole brain of the affine and deformable atlases. Figure 6 shows the gradient magnitude images and a histogram comparison. At the $90^{t h}$ percentile of the cumulative histogram, the deformable atlas has a gradient magnitude of 684 while the affine atlas is 573 , an increase of $20 \%$. This shows that the deformable atlas better preserves thin structures via improved alignment.

\section{Discussion and Future Work}

We have developed an automatic method for producing correspondence in diffusion tensor images through deformable registration, and a novel image match which alignes structural features. We apply the transformation using the finite strain model for tensor rotation and a Riemannian framework for averaging and interpolation. Initial validation of deformable registration is performed by showing improvement in thin structure preservation over affine alignment. 
Future validation work will try to quantify the quality of correspondence of fiber tracts. In future work, we intend to build DTI atlases of different populations to compare tract geometry and tensor statistics along tracts.

\section{References}

1. Corouge, I., Fletcher, P.T., Gilmore, J.H., Gerig, G.: Fiber tract-oriented statistics for quantitative diffusion tensor MRI analysis. In: MICCAI. Volume 3749 of LNCS. (2005) 131-139

2. Wakana, S., Jiang, H., Nagae-Poetscher, L.M., van Zijl, P.C.M., Mori, S.: Fiber Tract-based Atlas of Human White Matter Anatomy. Radiology 230(1) (2004) $77-87$

3. Joshi, S., Davis, B., Jomier, M., Gerig, G.: Unbiased diffeomorphic atlas construction for computational anatomy. NeuroImage (Supplemental issue on Mathematics in Brain Imaging) 23 (2004) S151-S160

4. Zhang, H., Yushkevich, P., Gee, J.: Registration of diffusion tensor images. In: CVPR. (2004)

5. Guimond, A., Guttmann, C.R.G., Warfield, S.K., Westin, C.F.: Deformable registration of dt-mri data based on transformation invariant tensor characteristics. In: ISBI. (2002)

6. Alexander, D., Pierpaoli, C., Basser, P., Gee, J.: Spatial transformations of diffusion tensor magnetic resonance images. IEEE Transactions on Medical Imaging 20(11) (2001)

7. Fletcher, P., Joshi, S.: Principal geodesic analysis on symmetric spaces: Statistics of diffusion tensors. In: ECCV 2004, Workshop CVAMIA. (2004) 87-98

8. Pennec, X., Fillard, P., Ayache, N.: A Riemannian framework for tensor computing. International Journal of Computer Vision 66(1) (2006) 41-66

9. Arsigny, V., Fillard, P., Pennec, X., Ayache, N.: Fast and simple calculus on tensors in the Log-Euclidean framework. In: MICCAI. Volume 3749 of LNCS. (2005) 115122

10. Westin, C.F., Maier, S.E., Mamata, H., Nabavi, A., Jolesz, F.A., Kikinis, R.: Processing and visualization of diffusion tensor MRI. Medical Image Analysis 6(2) (2002) 93-108 